\title{
Plasma Targeted Metabolomics Analysis for Amino Acids and Acylcarnitines in Patients with Prediabetes, Type 2 Diabetes Mellitus, and Diabetic Vascular Complications
}

\author{
Xin $\mathrm{Li}^{1}$, Yancheng $\mathrm{Li}^{2}$, Yuanhao Liang ${ }^{1}$, Ruixue $\mathrm{Hu}^{1}$, Wenli $\mathrm{Xu}^{1}$, Yufeng $\mathrm{Liu}^{1,3}$ \\ ${ }^{1}$ School of Pharmaceutical Sciences, Liaoning University, Shenyang, China, \\ ${ }^{2}$ Department of Biostatistics, College of Public Health and Health Professions \& College of Medicine, University of Florida, Gainesville, FL, USA, \\ ${ }^{3}$ Natural Products Pharmaceutical Engineering Technology Research Center of Liaoning Province, Shenyang, China
}

\begin{abstract}
Background: We hypothesized that specific amino acids or acylcarnitines would have benefits for the differential diagnosis of diabetes. Thus, a targeted metabolomics for amino acids and acylcarnitines in patients with diabetes and its complications was carried out. Methods: A cohort of 54 normal individuals and 156 patients with type 2 diabetes mellitus and/or diabetic complications enrolled from the First Affiliated Hospital of Jinzhou Medical University was studied. The subjects were divided into five main groups: normal individuals, impaired fasting glucose, overt diabetes, diabetic microvascular complications, and diabetic peripheral vascular disease. The technique of tandem mass spectrometry was applied to obtain the plasma metabolite profiles. Metabolomics multivariate statistics were applied for the metabolic data analysis and the differential metabolites determination.

Results: A total of 10 cross-comparisons within diabetes and its complications were designed to explore the differential metabolites. The results demonstrated that eight comparisons existed and yielded significant metabolic differences. A total number of 24 differential metabolites were determined from six selected comparisons, including up-regulated amino acids, down-regulated medium-chain and long-chain acylcarnitines. Altered differential metabolites provided six panels of biomarkers, which were helpful in distinguishing diabetic patients.

Conclusion: Our results demonstrated that the biomarker panels consisted of specific amino acids and acylcarnitines which could reflect the metabolic variations among the different stages of diabetes and might be useful for the differential diagnosis of prediabetes, overt diabetes and diabetic complications.
\end{abstract}

Keywords: Amino acids; Carnitine; Diabetes mellitus, type 2; Metabolomics

\section{INTRODUCTION}

Type 2 diabetes mellitus (T2DM) is a metabolic disorder with high morbidity and severe damages. According to the prevalence study in 2017 from the International Diabetes Federation [1], the population of patients with diabetes in world was about 450 million by 2017 , and this population would increase to 693 million in 2045. Moreover, T2DM can lead to various compli- cations, such as diabetic retinopathy, nephropathy, and diabetic vascular diseases [2].

However, there is no prefect medical means to cure diabetes and its complications till date. Currently, medical treatment is devoted to reduce and control the blood glucose of patients in the prevention of T2DM [3,4]. Positive intervention and treatment are both beneficial to slow down the progresses of diabetes. As for the methods stated above, an early and accurate di-

\footnotetext{
Corresponding author: Yufeng Liu (D) https://orcid.org/0000-0001-7972-8771 School of Pharmaceutical Sciences, Liaoning University, Zheli Rd, Huanggu District, Shenyang 110036, China

E-mail: liuyufeng@bjmu.edu.cn

Received: Nov. 6, 2019; Accepted: Feb. 26, 2020
}

This is an Open Access article distributed under the terms of the Creative Commons Attribution Non-Commercial License (https://creativecommons.org/licenses/by-nc/4.0/) which permits unrestricted non-commercial use, distribution, and reproduction in any medium, provided the original work is properly cited. 
agnosis is the most important step, which should be done before implementing any of the aforementioned medical cares.

Metabolomics is a powerful tool for investigating the metabolic changes and regulations of small molecules in the human body [5]. It has been widely used in the study of diseases, especially the discovery of disease biomarkers [5-7]. According to previous studies [8-12], the metabolic profiles of amino acids and acylcarnitines are close related to the occurrence and development of T2DM. And there are also a great number of studies that have demonstrated that these metabolites had the potential to be the differential biomarkers for the diagnosis of diabetes.

Tandem mass spectrometry is commonly used for new born screening due to the multianalyte detection approach, such as the profile measurement of disordered metabolites rather than a single analyte measurement [13]. Moreover, the application of ultra-performance liquid chromatography (UPLC) in metabolomics also represents an amount of advantages for the determination of endogenous low-molecular-weight metabolites [14]. Consequently, the UPLC coupled to tandem mass spectrometric technique (UPLC-MS/MS) has been recently applied to metabolic researches recently, especially for clinical metabolomics $[15,16]$.

Thus, a targeted metabolomics based on MS/MS for studying the T2DM and its complications was carried out for exploring the metabolic changes of amino acids and acylcarnitine as well as discovering the biomarkers for differential diagnosis.

\section{METHODS}

\section{Patients and experiments design}

Our study was approved by the Ethics Committee for Clinical Research of the First Affiliated Hospital of Jinzhou Medical University and the informed consent was waived due to the nature of the retrospective study, which was in accordance with the Helsinki Declaration of 1964 and its later amendments [17,18]. The approval number for IRB on our study is JZURLL20200001.

A cohort of 210 subjects (including 54 normal individuals [NIs], 32 impaired fasting glucoses [IFGs], 21 simple diabetes [SDs], and 103 diabetic complications [DCs]) was randomly recruited from the First Affiliated Hospital of Jinzhou Medical University from March 2016 to March 2017. They were between ages of 19 to 77 and not related to each other. There were no significant differences in the composition of gender and age among all control groups and disease groups according to the $t$-test $(P>0.05)$.

The patients did not take steroids or nonsteroidal anti-inflammatory drugs, lipid-regulating drugs and other drugs at least in 2 weeks prior to the treatment. The included standards of the patients were as follows: all patients were diagnosed as T2DM according to the diabetes diagnosis and treatment standard (2018) of American Diabetes Association [19].

The excluded criteria of the diabetic group were as follows: (1) patients with hyperuricemia; (2) patients with malignant tumors; (3) patients received chemotherapy within 1 year; (4) patients received transfusions within 5 days; (5) patients with malnutrition; (6) patients with inherited metabolic diseases; and (7) patients with intravenous infusion of amino acids within 3 days.

\section{Training set and validation set}

In this study, all subjects were divides into 10 cross-comparisons (Supplementary Fig. 1) according to the stages of diabetes and the types of DCs. For every cross-comparison, samples were divided into a training set and a validation set according to the ratio of 3 to 2 [20]. Then, the training set was used to establish the predicting model in the multivariate data analysis while the validation set was used to assess the model.

\section{Detection of metabolites by tandem mass spectrometry Samples and preparation}

In this study, the blood samples were taken from all subjects. MS/MS was used to detect and measure amino acids and carnitines. The total detections contained 58 metabolites including 23 amino acids, 35 acylcarnitines (Supplementary Table 1).

A paper disc of $3 \mathrm{~mm}$ was punched from the dried blood spots (DBS) filter paper, which was equal to $3.2 \mu \mathrm{L}$ of whole blood. Then, the paper discs were inserted into a well in Millipore MultiScreen HV 96-well plate (Carrigtohill, Ireland) and subjected to the extraction of metabolites. Briefly, each $100 \mu \mathrm{L}$ of working solution was added into a well including a DBS disc. The plate was shaken gently for 20 minutes at room temperature and centrifuged at $1,500 \times g$ for 2 minutes. The filtrate was gathered into a new flat-bottom 96-well plate. For every plate, quality control (QC) sample solutions contained two high-level and two low-level solutions were randomly assigned into four blank wells and analyzed as real samples to monitor the stability of the MS/MS analysis. The QC solutions and filtrate were dried under pure nitrogen gas at $50^{\circ} \mathrm{C}$. We derived each dried sample in $60 \mu \mathrm{L}$ 1-butanol and acetyl chloride mix- 
ture $(90: 10, \mathrm{v} / \mathrm{v})$ at $65^{\circ} \mathrm{C}$ for 20 minutes, and the derivatized solutions were dried under pure nitrogen gas at $50^{\circ} \mathrm{C}$ again. Finally, we added $100 \mu \mathrm{L}$ mobile phase solution into each well before analysis.

\section{Chemicals}

HPLC-grade acetonitrile (ACN) and HPLC-grade water were acquired from Thermo Fisher (Waltham, MA, USA). Acetyl chloride and 1-butanol were purchased from Sigma-Aldrich (St. Louis, MO, USA). Internal standard kits including eight labelled carnitine internal standards (catalogue number: NSKA) and 12 stable isotope-labelled amino acid (catalogue number: NSK-B) for absolute quantification were obtained from Cambridge Isotope Laboratories (Andover, MA, USA). The characteristics of these internal labels were listed in Supplementary Table 2. QC amino acids and carnitines mixed standards were purchased from Chromsystems (Grafelfing, Germany).

NSK-A and NSK-B were dissolved with $1 \mathrm{~mL}$ of methanol to get a stock solution (stored at $4^{\circ} \mathrm{C}$ ). The working solution for metabolite extraction was prepared by diluting the stock solution 100 times. The QC samples were processed as real samples and randomly inserted in the analysis queue.

\section{Tandem mass spectrometry}

MS/MS analysis was implemented on an AB SCIEX 4000 QTrap system (AB Sciex, Framingham, MA, USA) equipped with an electrospray ionization source in positive mode. The injected volume was $20 \mu \mathrm{L}$. A mixture of $80 \% \mathrm{ACN}$ aqueous solution was used as the mobile phase. The elution program was initiated with $0.2 \mathrm{~mL} / \mathrm{min}$, then decreased to $0.1 \mathrm{~mL} / \mathrm{min}$ within 0.08 minute and kept constant until to 1.5 minutes. Next, the flow rate increased to $0.2 \mathrm{~mL} / \mathrm{min}$ within 0.1 minute and held for 0.5 minute. The mass spectrometry ion spray voltage was set at $4.5 \mathrm{kV}$. Pressures of Ion Source Gas 1 and Gas 2 were all 35 psi. Curtain gas pressure was 20 psi. The auxiliary gas temperature was held at $350^{\circ} \mathrm{C}$. The raw data were collected by Analyst v1.6.0 software (AB Sciex). The quantification of individual metabolites was accomplished by ChemoView 2.0.2 software (AB Sciex) against different isotope standards.

\section{Metabolomics study}

\section{Data pre-processing}

All data analysis involved in this study were coded by $\mathrm{R}$ (v3.6.3) scripts and implemented in R Studio (v 1.1.423). A se- ries of pre-processing were implemented to the primary dataset to ensure the datasets as reasonable, including imputation of missing data, data centralization and data standardization. In addition, identification based on boxplot and principal component analysis was carried out to remove the outliers.

\section{Univariate analysis}

In order to identify differential metabolites among cross-comparisons, $P$ values calculated by Kruskal-Wallis test and adjusted by false discovery ratio of 58 metabolites detected by MS/MS were obtained from R scripts. Logarithm of fold change at a base of 2 was calculated to reveal the extents and directions of metabolism changes between the controls and patients.

\section{Multivariate data analysis}

A supervised analysis, orthogonal partial least squares discriminant analysis (OPLS-DA), was used to establish a classifier of training set from 10 comparisons. Then, the parameters of 10-fold cross-validation were used to assess these OPLS-DA models. Potential biomarkers could be determined by combining $P$ values and the variable importance in projection (VIP) of this classification model. Based on VIP of these models, a heatmap and partial least squares regression analysis were used to reflect the metabolic regulations and the separated ability of these classifiers, respectively. Then, the receiver operating characteristic (ROC) curve and the prediction of validation set were carried out to evaluate the differential diagnosis ability for these identified biomarker panels.

\section{Pathways analyses}

The metabolic pathway analysis for every biomarker panel was implemented by Metaboanalysis 4.0 platform [21]. Significant influenced pathways for every biomarker panel were plotted. Based on the Kyoto Encyclopedia of Genes and Genomes (KEGG) pathway database, a metabolic diagram of all differential metabolites was mapped to present the alternatives within metabolism of amino acids and acylcarnitines in the development of T2DM.

\section{RESULTS}

A total of 210 participants (including 54 NIs, 32 patients with IFG, 21 patients with SD, and 103 patients with various DCs) were enrolled. The subjects were divided into 10 cross-com- 
parisons (Supplementary Fig. 1) according to the different stages and complications of diabetes. Six cross-comparisons composed of NIs and diabetes patients had been selected and used for further analysis to obtain more valuable metabolites within populations in different diabetic phases.

\section{Baseline characteristics}

Baseline characteristics of all experimental participants were shown in Supplementary Table 3. There was a progressive increase in blood glucose across the groups from NI to IFG, IFG to SD, and SD to DC. Patients with IFG, SD, and DC had much higher body mass index than NI; and patients with SD also showed a significant elevated blood pressure (either systolic or diastolic) compared to NI and IFG. In addition, patients with diabetic peripheral vascular disease (DPVD; a type of diabetic macro vascular complications) underwent a longer duration of disease than those with diabetic microvascular complication (DMVC). All groups showed no significant difference in age and gender ratio.

\section{Multivariate statistical analysis}

The OPLS-DA method was applied to investigate the existence of metabolites disturbance in the 10 cross-comparisons. Among 10 OPLS-DA models, eight of them were built successfully. Clear differences were obtained from all these comparisons, but our analysis mainly focused on the following six models (Fig. 1): IFG versus NI, cumulative $\mathrm{R}^{2} \mathrm{Y}$ at 0.907 and $\mathrm{Q}^{2}$ at 0.822 ; $S D$ versus IFG, cumulative $\mathrm{R}^{2} \mathrm{Y}$ at 0.858 and $\mathrm{Q}^{2}$ at 0.428; DMVC versus SD, cumulative $\mathrm{R}^{2} \mathrm{Y}$ at 0.962 and $\mathrm{Q}^{2}$ at 0.738; DPVD versus SD, cumulative $R^{2} Y$ at 0.971 and $Q^{2}$ at 0.683 ; DMVC \& DPVD versus SD, cumulative $\mathrm{R}^{2} \mathrm{Y}$ at 0.971 and $\mathrm{Q}^{2}$ at 0.899; DMVC \& DPVD versus DMVC, cumulative $\mathrm{R}^{2} \mathrm{Y}$ at 0.946 and $\mathrm{Q}^{2}$ at 0.897 . OPLS-DA score plots of other two comparisons, including SD versus NI and DPVD versus DMVC, were represented in Supplementary Fig. 2.
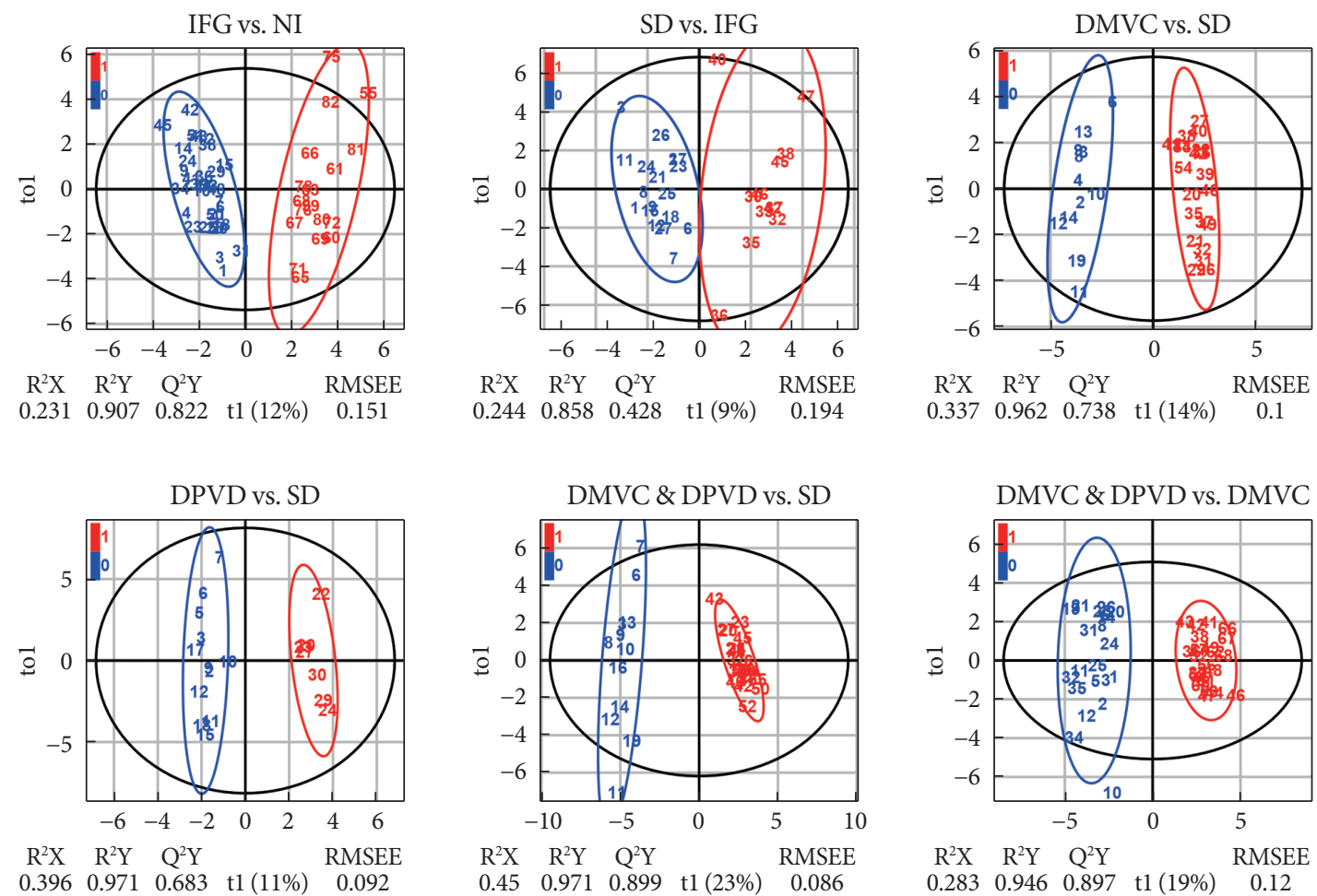

Fig. 1. The orthogonal partial least squares discriminant analysis (OPLS-DA) score plots were generated from six comparisons. In these score plots, individuals from control groups and comparable disease groups were represented by different colors ("blue" for control groups, "red" for case groups). The parameters of OPLS-DA models (including $R^{2} \mathrm{X}, \mathrm{R}^{2} \mathrm{Y}, \mathrm{Q}^{2} \mathrm{Y}$, and RMSEE) were also plotted to represent the quality of these established models. $t 1$ and tol were the first principal component and the first orthogonal component of OPLS-DA models, respectively. IFG, impaired fasting glucose; NI, normal individual; SD, simple diabetes; DMVC, diabetic microvascular complication; DPVD, diabetic peripheral vascular disease. 


\section{Differential metabolites and disturbed pathways}

Differential metabolites, including 11 amino acids and 13 acylcarnitines, with VIP $>1.5$ and adjusted $P$ values $<0.05$ from six paired comparisons were selected and listed in Table 1 (some metabolites not only appeared in one comparison). The heatmap in Fig. 2 represented the trends of all detected metabolites and differential metabolites in six groups, respectively.
Based on differential metabolites, the perturbed pathways were determined and shown in Fig. 3. For IFG versus NI, metabolic changes involved valine-leucine-isoleucine, fatty acid, and arginine-proline purine; for SD versus IFG, changes involved alanine-aspartate-glutamate, tryptophan, and nitrogen metabolism; for DMVC versus SD, changes involved alanineaspartate-glutamate, nitrogen, D-glutamine and D-glutamate

Table 1. The parameters of differential metabolites selected from six cross-comparisons

\begin{tabular}{|c|c|c|c|c|c|}
\hline Metabolites & VIP & $P$ value & $P$ adjusted & $\log _{2}$ (fold change) & AUC \\
\hline \multicolumn{6}{|l|}{ IFG vs. NI } \\
\hline C6DC & 2.676 & $2.10 \mathrm{E}-11$ & $1.22 \mathrm{E}-09$ & 3.282 & 0.950 \\
\hline $\mathrm{C} 14$ & 2.308 & $4.79 \mathrm{E}-10$ & $6.94 \mathrm{E}-09$ & 1.874 & 0.917 \\
\hline $\mathrm{C} 12$ & 2.246 & $1.43 \mathrm{E}-10$ & $2.76 \mathrm{E}-09$ & 4.086 & 0.930 \\
\hline $\mathrm{C} 14-\mathrm{OH}$ & 2.166 & $8.61 \mathrm{E}-11$ & $2.50 \mathrm{E}-09$ & 2.283 & 0.930 \\
\hline $\mathrm{C} 14: 1$ & 1.931 & $2.70 \mathrm{E}-08$ & $3.14 \mathrm{E}-07$ & 2.077 & 0.872 \\
\hline Leu & 1.786 & $1.25 \mathrm{E}-05$ & 7.23E-05 & -0.955 & 0.793 \\
\hline C16 & 1.750 & $1.69 \mathrm{E}-06$ & $1.40 \mathrm{E}-05$ & 1.129 & 0.821 \\
\hline $\mathrm{C} 14: 2$ & 1.702 & $5.15 \mathrm{E}-07$ & $4.98 \mathrm{E}-06$ & 1.507 & 0.837 \\
\hline Cit & 1.690 & $2.49 \mathrm{E}-06$ & $1.81 \mathrm{E}-05$ & -1.236 & 0.816 \\
\hline \multicolumn{6}{|l|}{ SD vs. IFG } \\
\hline Cit & 2.629 & $9.62 \mathrm{E}-08$ & $5.58 \mathrm{E}-06$ & 1.811 & 0.960 \\
\hline $\mathrm{C} 16: 1-\mathrm{OH}$ & 2.085 & $2.33 \mathrm{E}-05$ & $6.76 \mathrm{E}-04$ & -1.074 & 0.863 \\
\hline Try & 1.994 & $1.00 \mathrm{E}-04$ & $1.45 \mathrm{E}-03$ & 0.800 & 0.836 \\
\hline $\mathrm{C} 8$ & 1.820 & $4.30 \mathrm{E}-05$ & $8.32 \mathrm{E}-04$ & 2.055 & 0.852 \\
\hline Asn & 1.789 & $5.45 \mathrm{E}-04$ & $6.32 \mathrm{E}-03$ & 0.695 & 0.799 \\
\hline Leu & 1.575 & $2.32 \mathrm{E}-03$ & $1.92 \mathrm{E}-02$ & 0.998 & 0.763 \\
\hline \multicolumn{6}{|l|}{ DMVC vs. SD } \\
\hline C16 & 2.155 & $9.56 \mathrm{E}-08$ & $5.54 \mathrm{E}-06$ & -2.976 & 0.944 \\
\hline Asn & 1.866 & 7.61E-07 & $2.21 \mathrm{E}-05$ & -1.120 & 0.911 \\
\hline $\mathrm{C} 14: 2$ & 1.766 & $8.84 \mathrm{E}-04$ & $4.66 \mathrm{E}-03$ & -0.899 & 0.777 \\
\hline $\mathrm{C} 8$ & 1.634 & 3.97E-04 & $2.56 \mathrm{E}-03$ & -1.387 & 0.794 \\
\hline $\mathrm{C} 14$ & 1.607 & $2.57 \mathrm{E}-04$ & $1.86 \mathrm{E}-03$ & -1.499 & 0.803 \\
\hline C6DC & 1.586 & $1.70 \mathrm{E}-04$ & $1.65 \mathrm{E}-03$ & -0.856 & 0.813 \\
\hline Gln & 1.558 & $1.03 \mathrm{E}-05$ & $1.99 \mathrm{E}-04$ & -1.034 & 0.867 \\
\hline \multicolumn{6}{|l|}{ DPVD vs. SD } \\
\hline C6DC & 2.403 & $7.56 \mathrm{E}-06$ & $4.38 \mathrm{E}-04$ & -4.332 & 1.000 \\
\hline Orn & 2.330 & $5.15 \mathrm{E}-03$ & $3.32 \mathrm{E}-02$ & 1.550 & 0.813 \\
\hline Met & 2.020 & $1.16 \mathrm{E}-03$ & $1.81 \mathrm{E}-02$ & -1.386 & 0.864 \\
\hline $\mathrm{C} 14: 2$ & 1.945 & $3.25 \mathrm{E}-04$ & $9.42 \mathrm{E}-03$ & -2.070 & 0.902 \\
\hline C14DC & 1.857 & $4.13 \mathrm{E}-03$ & 2.99E-02 & -1.118 & 0.818 \\
\hline Try & 1.736 & $1.25 \mathrm{E}-03$ & $1.81 \mathrm{E}-02$ & -1.172 & 0.861 \\
\hline $\mathrm{C} 18-\mathrm{OH}$ & 1.575 & $2.94 \mathrm{E}-03$ & $2.45 \mathrm{E}-02$ & -1.640 & 0.828 \\
\hline
\end{tabular}


Table 1. Continued

\begin{tabular}{|c|c|c|c|c|c|}
\hline Metabolites & VIP & $P$ value & $P$ adjusted & $\log _{2}$ (fold change) & AUC \\
\hline \multicolumn{6}{|c|}{ DPVD \& DMVC vs. SD } \\
\hline Gln & 1.881 & $1.25 \mathrm{E}-09$ & $3.62 \mathrm{E}-08$ & -3.783 & 1.000 \\
\hline C6DC & 1.852 & $1.24 \mathrm{E}-09$ & 3.62E-08 & -2.965 & 1.000 \\
\hline Hcy & 1.677 & $5.24 \mathrm{E}-08$ & $7.60 \mathrm{E}-07$ & -5.112 & 0.948 \\
\hline Phe & 1.618 & $8.28 \mathrm{E}-09$ & $1.60 \mathrm{E}-07$ & -2.934 & 0.974 \\
\hline Tyr & 1.590 & $8.11 \mathrm{E}-08$ & $8.53 \mathrm{E}-07$ & -1.951 & 0.942 \\
\hline C14:2 & 1.506 & $6.16 \mathrm{E}-07$ & 3.97E-06 & -2.039 & 0.910 \\
\hline \multicolumn{6}{|c|}{ DPVD \& DMVC vs. DMVC } \\
\hline Hcy & 2.008 & $6.05 \mathrm{E}-12$ & $2.10 \mathrm{E}-10$ & 0.384 & 0.972 \\
\hline Cys & 1.898 & $1.09 \mathrm{E}-11$ & $2.10 \mathrm{E}-10$ & 0.236 & 0.966 \\
\hline $\mathrm{C} 16$ & 1.720 & $1.05 \mathrm{E}-11$ & $2.10 \mathrm{E}-10$ & -0.281 & 0.966 \\
\hline $\mathrm{C} 22$ & 1.595 & $9.99 \mathrm{E}-11$ & $1.16 \mathrm{E}-09$ & 0.147 & 0.941 \\
\hline $\mathrm{C} 18$ & 1.556 & $1.79 \mathrm{E}-10$ & $1.55 \mathrm{E}-09$ & 0.677 & 0.937 \\
\hline Phe & 1.521 & $1.87 \mathrm{E}-10$ & $1.55 \mathrm{E}-09$ & 0.432 & 0.937 \\
\hline
\end{tabular}

VIP, variable importance in projection; AUC, area under curve; IFG, impaired fasting glucose; NI, normal individual; SD, simple diabetes; DMVC, diabetic microvascular complication; DPVD, diabetic peripheral vascular disease.

metabolism; for DPVD versus SD, changes involved cysteinemethionine, arginine-proline and tryptophan metabolism; for DMVC \& DPVD versus SD, changes involved alanine-aspartate-glutamate, cysteine-methionine, phenylalanine and sulfur metabolism; for DMVC \& DPVD versus DMVC, changes involved cysteine-methionine, phenylalanine, and sulfur metabolism. Differential metabolites and disturbances of metabolism for other two comparisons could be found in Supplementary Table 4 and Supplementary Fig. 2.

\section{Potential biomarkers for differential diagnosis}

As shown in Table 1, six panels of differential biomarkers were generated from each cross-comparison, including nine metabolites for distinguishing IFG from NI, six for SD versus IFG, seven for DMVC versus SD, seven for DPVD versus SD, six for DMVC \& DPVD versus SD, and six for DMVC \& DPVD versus DMVC. The differential biomarkers for other comparisons were provided in Supplementary Table 4.

ROC and prediction of validation set were carried out to evaluate the potential of differential metabolites for predicting the diagnosis. ROC curve and prediction results were shown in Fig. 4. The areas under curves for six comparisons were 0.978 , $0.922,0.939,0.978,1.000$, and 1.000 in order of IFG versus NI, SD versus IFG, DMVC versus SD, DPVD versus SD, DMVC \& DPVD versus SD, and DMVC \& DPVD versus DMVC.
The predictions of both the training and validation sets were implemented based on OPLS regression method. The prediction plots of each comparison all demonstrated clear separation of disease groups from non-disease groups or complication groups from non-complications groups. The ROC and prediction plots for other two groups were represented in Supplementary Fig. 2.

\section{DISCUSSION}

Although the diagnosis of diabetes or prediabetes can currently be accomplished by a simple measurement of blood glucose, but there are also several reasons for supporting metabolite checks in our body at the same time. Firstly, the diagnosis of diabetes or prediabetes by the only one measurement-plasma glucose-may generate a false positive result and lead to a wrong diagnosis by the doctor. In this case, metabolites indexes may be a helpful assistant indicator. Secondly, these metabolites can also provide much more information about a diabetes or prediabetes patients. For example, the differential diagnosis metabolites might be able to tell us the progress of diseases. These will be valuable for providing the patients with a more specific and better treatment. Therefore, it is necessary to measure and assess the metabolites of diabetes and prediabetes patients.

This research investigated the metabolic disturbances of 

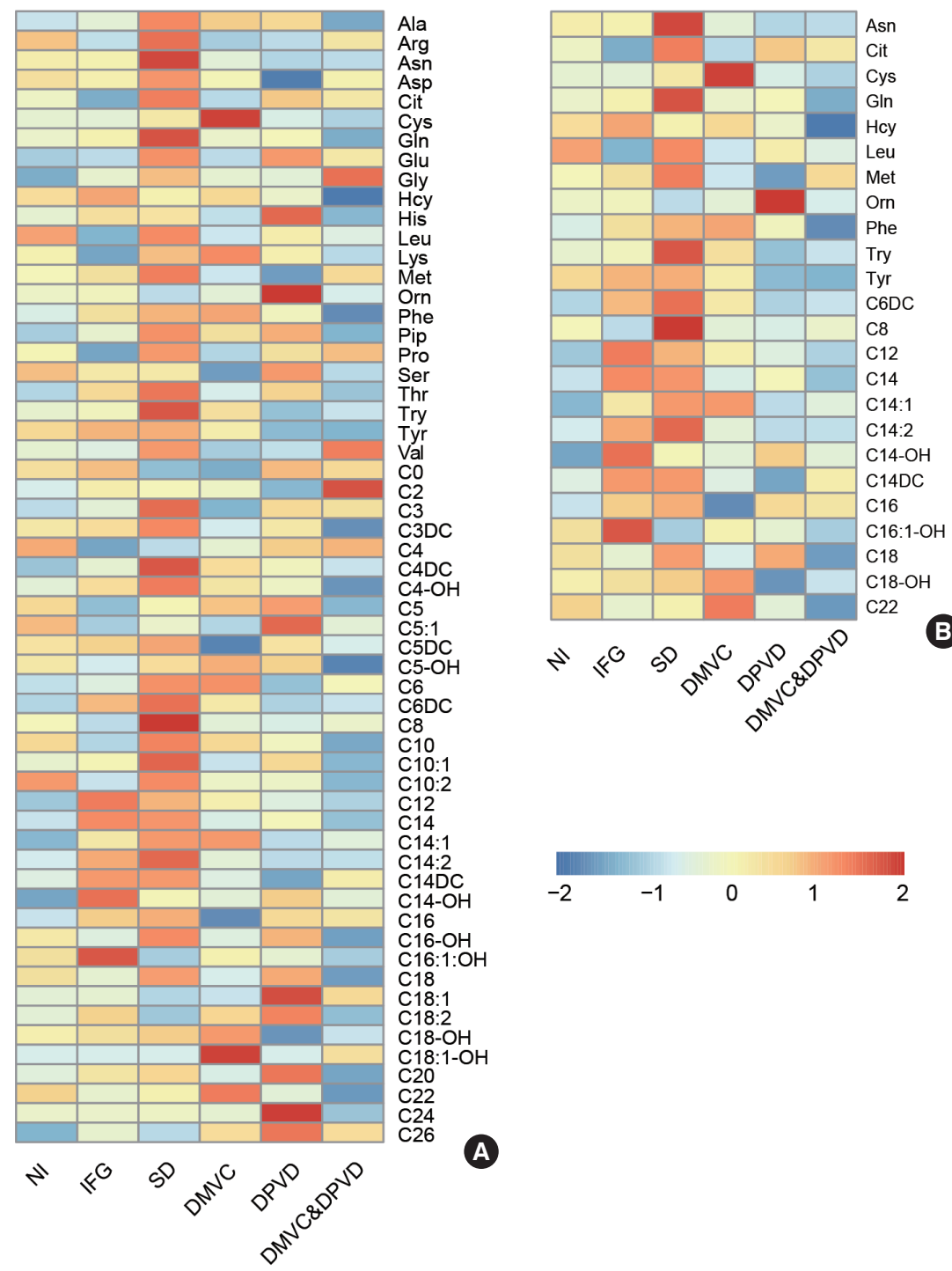

○

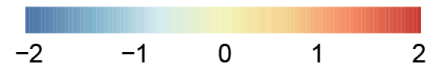

Fig. 2. Differential metabolites were determined by six comparisons. (A) The heatmap represented the levels of all 58 metabolites in six groups and (B) the heatmap represented the level of 24 differential metabolites in six groups. NI, normal individual; IFG, impaired fasting glucose; SD, simple diabetes; DMVC, diabetic microvascular complication; DPVD, diabetic peripheral vascular disease.

amino acids and acylcarnitines among NIs, patients with IFG, patients with SD and patients with various diabetic vascular complications. Metabolic phenotypes revealed that there were significant differences within the metabolism of normal persons, IFG, diabetes and DCs. The results of our study proved that amino acids and acylcarnitines were of great association with diabetes development and further attempted to clear these relevant metabolites. In addition, the results also provided a series of metabolite panels that might be benefit for the early prediction of T2DM and the differential diagnosis of
DCs. Specifically, these panels might offer valuable assistances for the prediagnosis, prevention, and prognosis of diabetes, such as graded prevention and treatment of diabetes.

\section{Comparisons within normal, impaired fasting glucose, and simple diabetes}

IFG is considered to be an intermediate states of glucose metabolism appeared between normal and overt diabetes, which is usually caused by relatively deficiency of insulin secretion (such as low insulin sensitivity and injury of islet $\beta$-cell func- 
IFG vs. NI

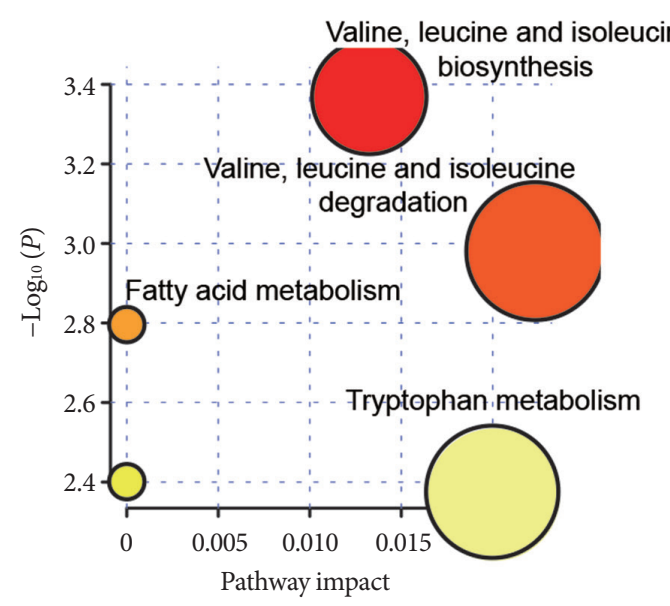

DMVC vs. SD

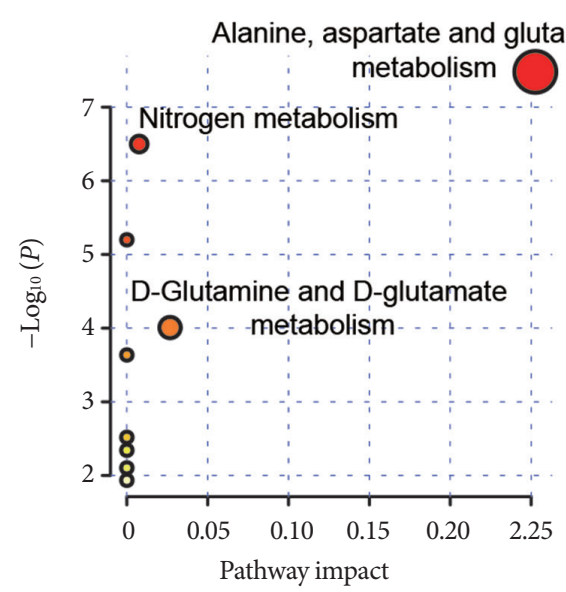

DMVC \& DPVD vs. SD

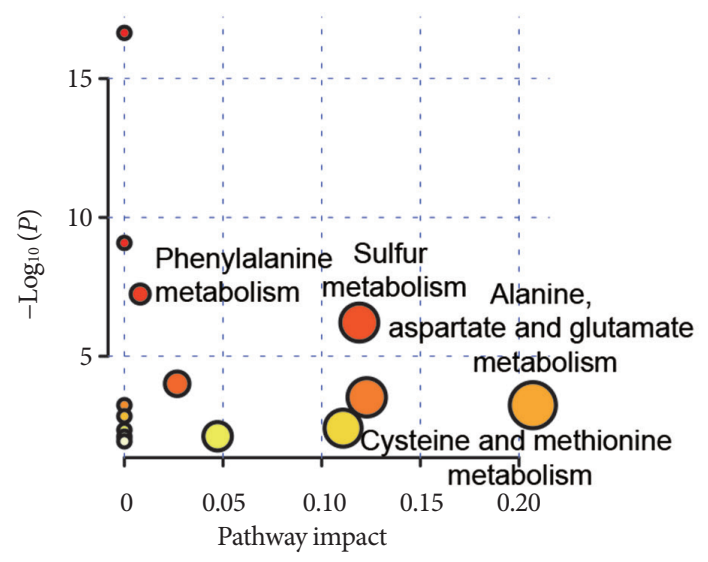

SD vs. IFG

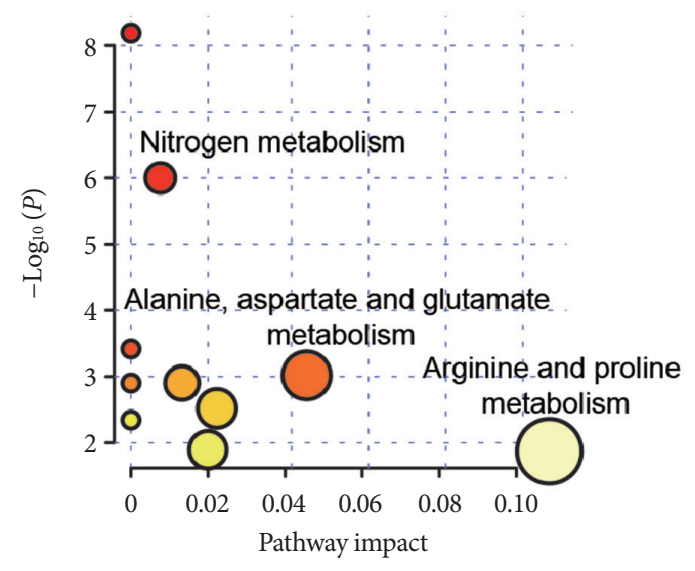

DPVD vs. SD

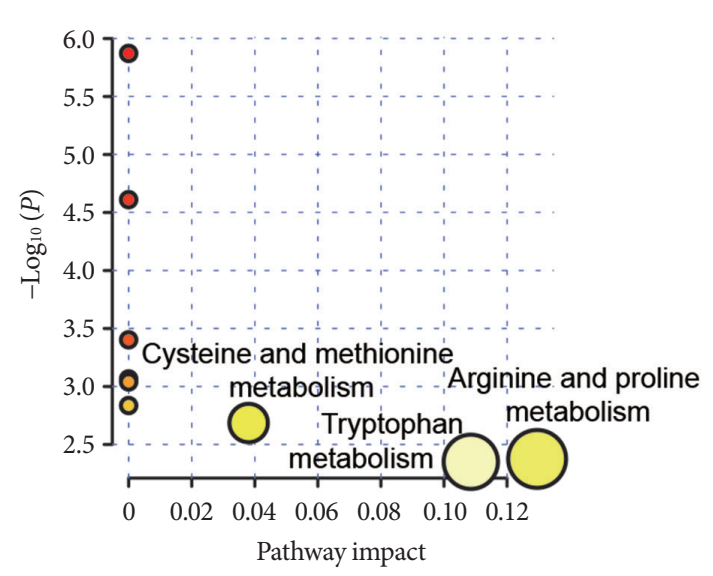

DMVC \& DPVD vs. DMVC

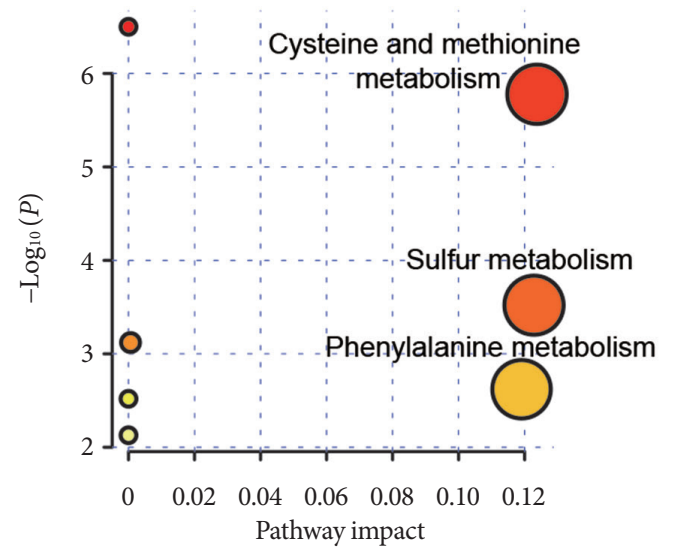

Fig. 3. The enrichment pathways of each biomarker were provided by Metaboanalysis 4.0 platform. IFG, impaired fasting glucose; NI, normal individual; SD, simple diabetes; DMVC, diabetic microvascular complication; DPVD, diabetic peripheral vascular disease. 
IFG vs. NI

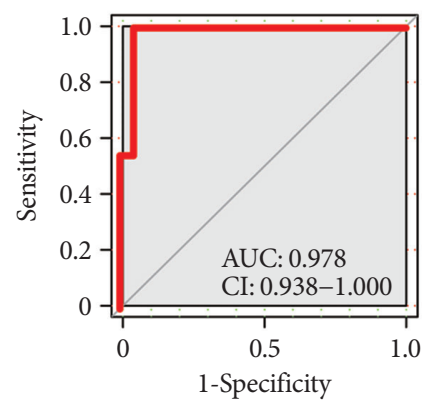

DPVD vs. SD

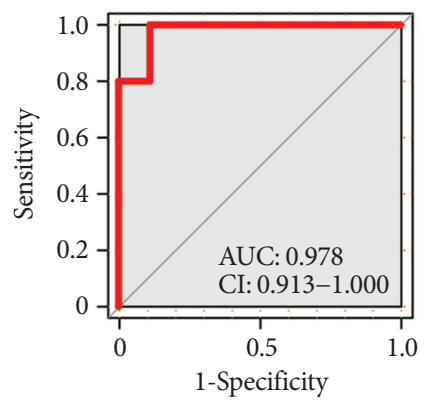

IFG vs. NI

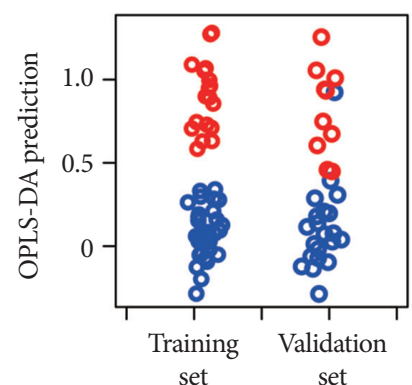

DPVD vs. SD

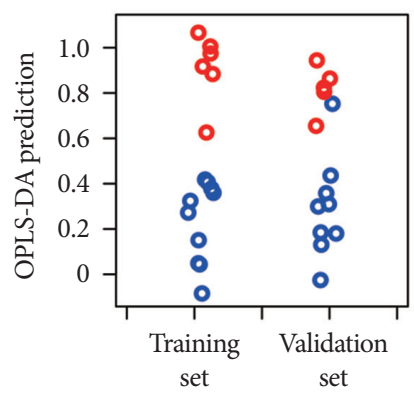

SD vs. IFG

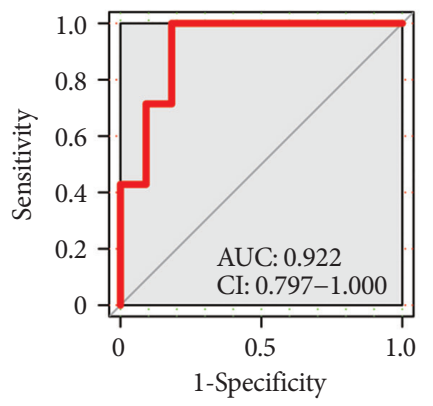

DMVC \& DPVD vs. SD

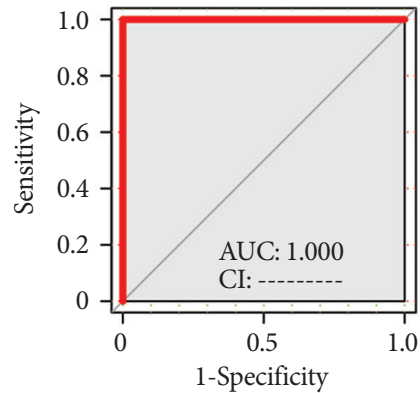

SD vs. IFG

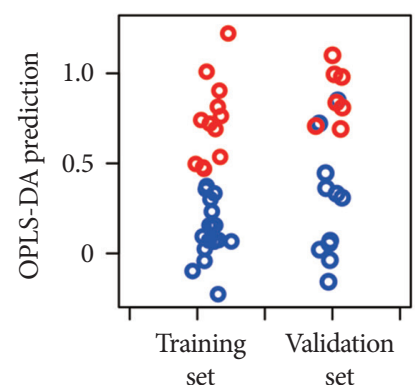

DMVC \& DPVD vs. SD

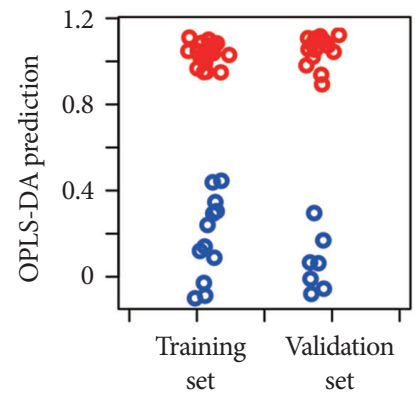

DMVC vs. SD

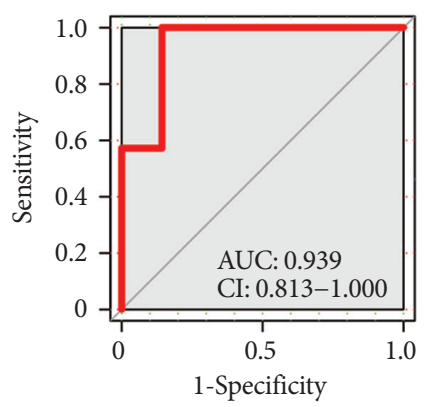

DMVC \& DPVD vs. DMVC

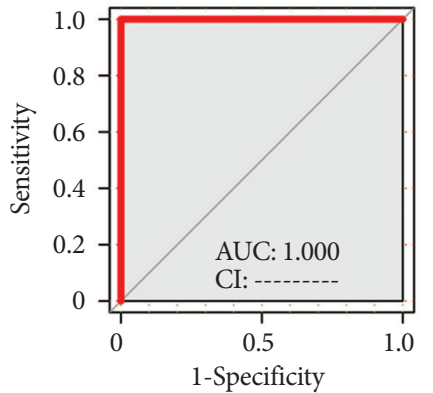

DMVC vs. SD

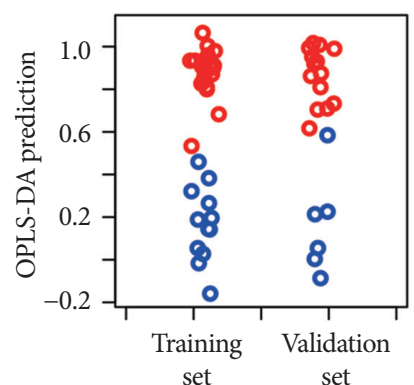

DMVC \& DPVD vs. DMVC

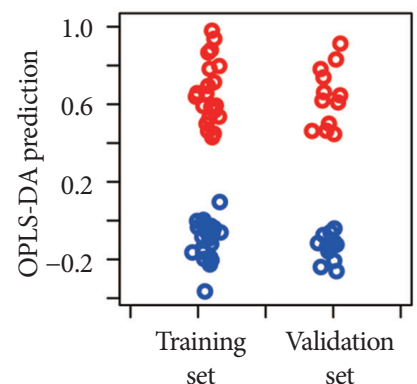

Fig. 4. The receiver operating characteristic (ROC) curves and prediction plots using the model were established by the six biomarker panels. These plots were plotted to represent the sensitivity, specify and predictive capacity of the partial least square (PLS) regression models established by each biomarker panel. In the ROC curves, the area under curve (AUC) and confidence interval (CI) were also given in the plots. In the prediction plots, individuals from control group and case group were also represented by different colors (blue and red). IFG, impaired fasting glucose; NI, normal individual; SD, simple diabetes; DMVC, diabetic microvascular complication; DPVD, diabetic peripheral vascular disease. 

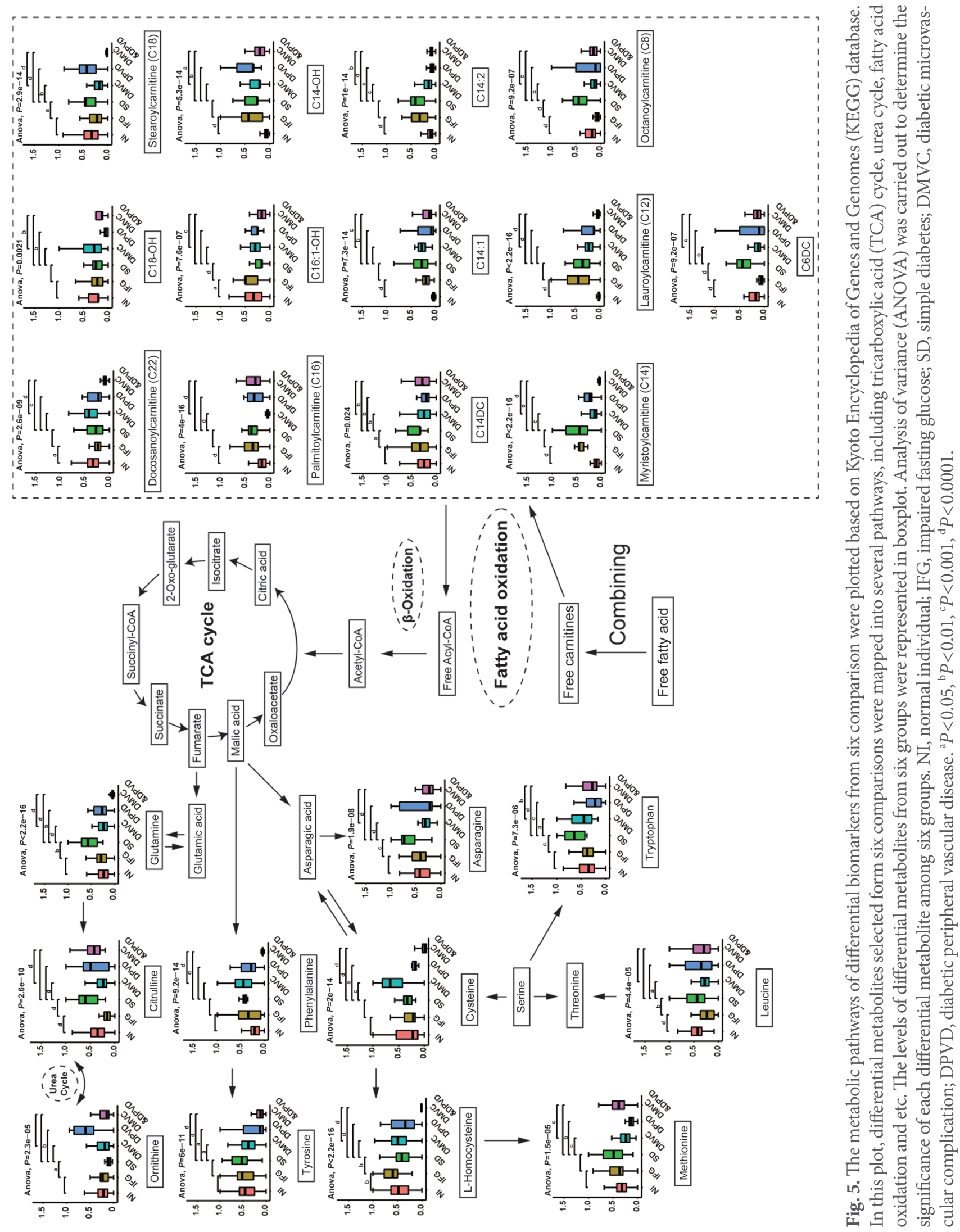
tion) [22,23]. During this period, patients with IFG usually have a great chance to reduce blood glucose to normal by reasonable dietary control and regular exercise interventions [24]. So, the early detection and diagnosis were crucial for population with potential diabetes. In our studies, comparing with NIs, patients with IFG showed down-regulated leucine (Leu), citrulline (Cit), C16; and up-regulated C6DC, C12, C14, C14:1, C14:2, C14-OH. While comparing with IFG patients, simple diabetic patients represented increased Cit, tyrosine (Try), asparagine (Asn), Leu, C8, and only decreased C16:1-OH.

According to the previous studies $[9,10,25]$, Leu is proved to be able to stimulate insulin secretion by activating the mammalian target of rapamycin signaling pathways and trends to accumulate in populations with prospective insulin resistance or T2DM. In our research, significant increased Leu in patients with overt T2DM proved the previous conclusion [26]. However, the level of Leu was decreased in patients with IFG, which were never reported in any similar studies. We supposed that it might be a new early characteristic of transition from NIs to prediabetes, although this also might be caused by the individual differences of patients, which need to be further validated in a bigger cohort study.

As shown in Fig. 5, Cit is an essential metabolite in urea cycle and associated with tricarboxylic acid (TCA) cycle through Lglutamine [12]. In the comparison of IFG versus NI, Cit showed a significant decrease in IFG patients, which was consistent to the alternatives of Leu. While in overt diabetic patients, Cit represented a significant elevation comparing with IFG patients, which might result from the elevation of L-glutamine in diabetes [27].

Asn and Try were the other two differential amino acids in the comparison of SD versus IFG distinct from that of IFG versus NI. They were involved in the alanine-aspartate-glutamate metabolism and tryptophan metabolism, respectively. Recently, in a 5.5 years follow-up study, higher level of Asn was proved to predict a progression to prediabetes [28]. Although, in our research, Asn level did not represent a significant increase between NIs and IFG patients, it showed a significant high level in patients with diabetes comparing to patients with IFG. Moreover, Yu et al. [29] and Matsuoka et al. [30] both revealed that tryptophan and its metabolites were both associated with higher risk of incident T2DM in a Prevención con Dieta Mediterránea (PREDIMED) trial.

Free fatty acids are another important source of acetyl coenzyme A (acetyl-CoA) for TCA cycle other than that of glucose, but an important prerequisite is that they must be esterified to free carnitines to form acylcarnitines that can then be transported into the mitochondria to be further oxidized [31]. However, the incomplete oxidation of long-chain fatty acids (LCFAs) in patients would result in the accumulation of acylcarnitines [31]. The studies revealed that the medium- and long-chain acylcarnitines tended to increase in patients with IFG as well as diabetic patients, which were probably associated with the incomplete combustion of LCFAs [32,33]. In addition, the inefficient LCFA beta-oxidation might also increase the accumulation of acetyl-CoA and generate short chain acylcarnitines that could activate proinflammatory pathways to exacerbate insulin resistance [33,34].

\section{Comparisons within different diabetic complications}

Patients with a longer history of diabetes usually represented various complications as well as more differential metabolisms [35]. In the cross-comparisons within SD and different DCs, there were either similarities or subtle distinctions in differential metabolites. For the comparison of DMVC versus SD, individuals with DMVC showed down-regulated both amino acids and acylcarnitines, including Asn, Gln, C6DC, C8, C14, C14:2, and C16. For the comparison of DPVD versus SD, patients with DPVD, showed up-regulated Orn and down-regulated C6DC, Met, C14:2, C14DC, Try, C18-OH. And for the comparison of DMVC \& DPVD versus SD, C6DC and C14DC showed down-regulated; while the other differential metabolites, including Gln, Hcy, Phe, Tyr, were significantly decreased.

Based on the changes of these differential metabolites, we could conclude that patients with DMVC or DPVD or with both usually represented the obviously decreased amino acids and acylcarnitines. However, there were also several abnormal amino acids and acylcarnitines, such as Orn and C14:1, represented diverse tendency among the group of DMVC, DPVD, and DMVC \& DPVD. Although other metabolites also showed those diverse directions which were controversial considering the pathophysiology of DCs, we supposed that the abnormal trend of these biomarkers might be the potential feature of the patients with the DMVC and DPVD at the same time. For these reasons, it is necessary to include much more patients with the similar conditions to perform more validation analysis.

For the changes of amino acids in diabetic micro and macro vascular complications, a recent case-cohort study attempted to explore the relationships within the circulating amino acids and the risk of diabetic vascular complications. In this study, 
the higher phenylalanine, lower glutamine and histidine were associated with an increased macrovascular risk; while the higher Try and alanine was associated with the decreased risk of microvascular [32]. In our study, Gln, Phe, and Tyr represented significant different among SD, DMVCs, and diabetic macrovascular complications. There also existed several differential amino acids that were first reported. Although these might be novel characteristics for the prediction of DCs, they also needed to be further validated.

There were only a few studies that reported the correlations between the changes of acylcarnitines and the occurrence of diabetic vascular complications. For example, in a recent study focused on kidney disease progression due to T2DM in American Indians, the results also suggested that patients with progressive pattern had a lower abundance of long-chain acylcarnitines (C16-20) [36]. In our studies, we assumed that the decreased amino acids and acylcarnitines in patients with DCs might be related to the compensation of patients during a long-term disease, such as the enhanced TCA cycle might be used to supply energy for human bodies. Meanwhile, we believed that the metabolism in diabetic patients would even worsen along with the prolongation of the clinical course.

In addition, we supposed that there might also exist some kinds of differential metabolites between diabetic microvascular disease and macrovascular disease. Thus, the comparison of DMVC \& DPVD versus DMVC was analyzed. We found that long chain acylcarnitines and amino acids were both significantly decreased in patients with microvascular and macrovascular disease compared to patients with microvascular alone, which also revealed the same metabolic pattern aforementioned in comparison with patients having DCs and SD.

The current research was the first to implement such a comprehensive diabetic study for investigating the differential metabolism of NIs and patients in different disease status. Metabolism changes were observed from six cross-comparisons, and six biomarker panels composed of specific amino acids and acylcarnitines were obtained from all differential metabolites. These biomarkers were of great significance to the differential diagnosis and graded prevention of diabetes in clinical studies. Specifically, those biomarkers from the comparison of IFG versus NI and SD versus IFG might be an assistant for the early diagnosis of diabetes, which were probably used to be the risk factors for the primary or secondary prevention of diabetes; while the biomarkers from the other four comparisons were of great ability to distinguish different DCs from SD, which might be the excellent prediction biomarkers for the prognosis of diabetic, as well as tertiary prevention.

In conclusion, in this study, we proved that amino acids and acylcarnitines could distinguish NIs, IFG, SD, and DCs by metabolomics based on MS/MS. Six biomarkers panels that could be used for the differential diagnosis of diabetes and DCs were identified and represented excellent predicting abilities. Based on our results, these differential biomarkers might be a meaningful supplement for the early diagnosis, prevention, and prognosis of T2DM. Moreover, the investigations of the metabolic profiles of differential amino acids and acylcarnitines were of great potency for understanding the development of diabetes and its complications.

\section{SUPPLEMENTARY MATERIALS}

Supplementary materials related to this article can be found online at https://doi.org/10.4093/dmj.2019.0209.

\section{CONFLICTS OF INTEREST}

No potential conflict of interest relevant to this article was reported.

\section{AUTHOR CONTRIBUTIONS}

Conception or design: X.L., Y.L.

Acquisition, analysis, or interpretation of data: X.L., Y.L., R.H., W.X., Y.L.

Drafting the work or revising: X.L., Y.L., R.H., W.X.

Final approval of the manuscript: Y.L., Y.L.

\section{ORCID}

Xin Li https://orcid.org/0000-0001-7717-8892

Yufeng Liu https://orcid.org/0000-0001-7972-8771

\section{FUNDING}

This work was supported by the National Natural Science Foundation of China (No. 81403177), the Science and Technology Planning Project of Shenyang Science and Technology Bureau (No. F12-277-1-14), and the Innovative Talents Support Program of Liaoning Province (LR2018047). 


\section{ACKNOWLEDGMENTS}

The authors thank all doctors, nurses and research staff at the First Affiliated Hospital of Jinzhou Medical University, for their participation in this study.

\section{REFERENCES}

1. Ogurtsova K, da Rocha Fernandes JD, Huang Y, Linnenkamp U, Guariguata L, Cho NH, et al. IDF diabetes atlas: global estimates for the prevalence of diabetes for 2015 and 2040. Diabetes Res Clin Pract 2017;128:40-50.

2. Forbes JM, Fotheringham AK. Vascular complications in diabetes: old messages, new thoughts. Diabetologia 2017;60:212938.

3. Fowler MJ. Microvascular and macrovascular complications of diabetes. Clin Diabetes 2008;26:77-82.

4. American Diabetes Association. 15. Diabetes care in the hospital: standards of medical care in diabetes-2019. Diabetes Care 2019;42:S173-81.

5. Jacob M, Lopata AL, Dasouki M, Abdel Rahman AM. Metabolomics toward personalized medicine. Mass Spectrom Rev 2019;38:221-38.

6. Guasch-Ferre M, Hruby A, Toledo E, Clish CB, MartinezGonzslez MA, Salas-Salvado J, et al. Metabolomics in prediabetes and diabetes: a systematic review and meta-analysis. Diabetes Care 2016;39:833-46.

7. Klein MS, Shearer J. Metabolomics and type 2 diabetes: translating basic research into clinical application. J Diabetes Res 2016;2016:3898502.

8. Batch BC, Hyland K, Svetkey LP. Branch chain amino acids: biomarkers of health and disease. Curr Opin Clin Nutr Metab Care 2014;17:86-9.

9. Giesbertz P, Daniel H. Branched-chain amino acids as biomarkers in diabetes. Curr Opin Clin Nutr Metab Care 2016;19: 48-54.

10. Lynch CJ, Adams SH. Branched-chain amino acids in metabolic signalling and insulin resistance. Nat Rev Endocrinol 2014;10:723-36.

11. Sun L, Liang L, Gao X, Zhang H, Yao P, Hu Y, et al. Early prediction of developing type 2 diabetes by plasma acylcarnitines: a population-based study. Diabetes Care 2016;39:1563-70.

12. Bi X, Henry CJ. Plasma-free amino acid profiles are predictors of cancer and diabetes development. Nutr Diabetes 2017;7: e249.
13. Chace DH, Kalas TA. A biochemical perspective on the use of tandem mass spectrometry for newborn screening and clinical testing. Clin Biochem 2005;38:296-309.

14. Zhao YY, Cheng XL, Vaziri ND, Liu S, Lin RC. UPLC-based metabonomic applications for discovering biomarkers of diseases in clinical chemistry. Clin Biochem 2014;47:16-26.

15. Ceglarek U, Leichtle A, Brugel M, Kortz L, Brauer R, Bresler K, et al. Challenges and developments in tandem mass spectrometry based clinical metabolomics. Mol Cell Endocrinol 2009; 301:266-71.

16. Denoroy L, Zimmer L, Renaud B, Parrot S. Ultra high performance liquid chromatography as a tool for the discovery and the analysis of biomarkers of diseases: a review. J Chromatogr B Analyt Technol Biomed Life Sci 2013;927:37-53.

17. Human experimentation: code of ethics of the world medical association (Declaration of Helsinki). Can Med Assoc J 1964; 91:619.

18. World Medical Association. World Medical Association Declaration of Helsinki: ethical principles for medical research involving human subjects. JAMA 2013;310:2191-4.

19. American Diabetes Association. 2. Classification and diagnosis of diabetes: standards of medical care in diabetes-2018. Diabetes Care 2018;41:S13-27.

20. Chaudhary K, Poirion OB, Lu L, Garmire LX. Deep learningbased multi-omics integration robustly predicts survival in liver cancer. Clin Cancer Res 2018;24:1248-59.

21. Chong J, Soufan O, Li C, Caraus I, Li S, Bourque G, et al. MetaboAnalyst 4.0: towards more transparent and integrative metabolomics analysis. Nucleic Acids Res 2018;46:W486-94.

22. Yip WCY, Sequeira IR, Plank LD, Poppitt SD. Prevalence of pre-diabetes across ethnicities: a review of impaired fasting glucose (IFG) and impaired glucose tolerance (IGT) for classification of dysglycaemia. Nutrients 2017;9:1273.

23. Abdul-Ghani MA, Tripathy D, DeFronzo RA. Contributions of beta-cell dysfunction and insulin resistance to the pathogenesis of impaired glucose tolerance and impaired fasting glucose. Diabetes Care 2006;29:1130-9.

24. Yang XJ, Zou SF, Xu Y, Li Y, Yang SS. The influence of intensive lifestyle intervention on patients with isolated impaired fasting glucose: a meta-analysis. J Adv Nurs 2016;72:2587-97.

25. Chen T, Ni Y, Ma X, Bao Y, Liu J, Huang F, et al. Branchedchain and aromatic amino acid profiles and diabetes risk in Chinese populations. Sci Rep 2016;6:20594.

26. Xu F, Tavintharan S, Sum CF, Woon K, Lim SC, Ong CN. Metabolic signature shift in type 2 diabetes mellitus revealed by 
mass spectrometry-based metabolomics. J Clin Endocrinol Metab 2013;98:E1060-5.

27. Ottosson F, Smith E, Melander O, Fernandez C. Altered asparagine and glutamate homeostasis precede coronary artery disease and type 2 diabetes. J Clin Endocrinol Metab 2018;103: 3060-9.

28. Owei I, Umekwe N, Stentz F, Wan J, Dagogo-Jack S. Amino acid signature predictive of incident prediabetes: a case-control study nested within the longitudinal pathobiology of prediabetes in a biracial cohort. Metabolism 2019;98:76-83.

29. Yu E, Papandreou C, Ruiz-Canela M, Guasch-Ferre M, Clish $\mathrm{CB}$, Dennis C, et al. Association of tryptophan metabolites with incident type 2 diabetes in the PREDIMED trial: a casecohort study. Clin Chem 2018;64:1211-20.

30. Matsuoka K, Kato K, Takao T, Ogawa M, Ishii Y, Shimizu F, et al. Concentrations of various tryptophan metabolites are higher in patients with diabetes mellitus than in healthy aged male adults. Diabetol Int 2016;8:69-75.

31. McCoin CS, Knotts TA, Adams SH. Acylcarnitines: old actors auditioning for new roles in metabolic physiology. Nat Rev Endocrinol 2015;11:617-25.
32. Batchuluun B, Al Rijjal D, Prentice KJ, Eversley JA, Burdett E, Mohan H, et al. Elevated medium-chain acylcarnitines are associated with gestational diabetes mellitus and early progression to type 2 diabetes and induce pancreatic $\beta$-cell dysfunction. Diabetes 2018;67:885-97.

33. Adams SH, Hoppel CL, Lok KH, Zhao L, Wong SW, Minkler $\mathrm{PE}$, et al. Plasma acylcarnitine profiles suggest incomplete long-chain fatty acid beta-oxidation and altered tricarboxylic acid cycle activity in type 2 diabetic African-American women. J Nutr 2009;139:1073-81.

34. Rutkowsky JM, Knotts TA, Ono-Moore KD, McCoin CS, Huang S, Schneider D, et al. Acylcarnitines activate proinflammatory signaling pathways. Am J Physiol Endocrinol Metab 2014;306:E1378-87.

35. Zheng Y, Ley SH, Hu FB. Global aetiology and epidemiology of type 2 diabetes mellitus and its complications. Nat Rev Endocrinol 2018;14:88-98.

36. Afshinnia F, Nair V, Lin J, Rajendiran TM, Soni T, Byun J, et al. Increased lipogenesis and impaired $\beta$-oxidation predict type 2 diabetic kidney disease progression in American Indians. JCI Insight 2019;4:e130317. 
Supplementary Table 1. The statistics of the concentration of metabolites in different case groups

\begin{tabular}{|c|c|c|c|c|c|c|}
\hline Metabolites & NI & IFG & $\mathrm{SD}$ & DMVC & DPVD & DMVC \& DPVD \\
\hline Ala & $132.20 \pm 57.29$ & $125.83 \pm 50.77$ & $121.97 \pm 39.53$ & $151.44 \pm 49.87$ & $153.98 \pm 34.03$ & $152.22 \pm 48.39$ \\
\hline Arg & $7.01 \pm 2.82$ & $5.29 \pm 3.12$ & $6.07 \pm 2.96$ & $9.57 \pm 5.45$ & $8.69 \pm 5.39$ & $8.46 \pm 4.47$ \\
\hline Asn & $54.02 \pm 15.82$ & $56.08 \pm 14.73$ & $44.57 \pm 11.23$ & $68.79 \pm 26.42$ & $67.33 \pm 20.62$ & $69.54 \pm 16.66$ \\
\hline Asp & $28.89 \pm 10.04$ & $25.40 \pm 10.73$ & $27.65 \pm 11.96$ & $29.59 \pm 11.04$ & $25.62 \pm 8.50$ & $31.37 \pm 11.98$ \\
\hline Cit & $21.26 \pm 6.54$ & $19.95 \pm 9.80$ & $17.32 \pm 5.00$ & $24.15 \pm 8.94$ & $27.73 \pm 11.61$ & $24.77 \pm 10.31$ \\
\hline Cys & $1.27 \pm 0.80$ & $0.95 \pm 0.83$ & $0.90 \pm 0.51$ & $1.11 \pm 0.46$ & $0.90 \pm 0.84$ & $1.31 \pm 1.49$ \\
\hline Gln & $9.29 \pm 4.27$ & $7.31 \pm 3.31$ & $7.74 \pm 2.91$ & $7.99 \pm 4.41$ & $7.46 \pm 3.51$ & $12.32 \pm 21.94$ \\
\hline Glu & $121.52 \pm 40.25$ & $129.49 \pm 46.54$ & $109.22 \pm 32.74$ & $130.02 \pm 47.28$ & $114.06 \pm 35.65$ & $136.50 \pm 42.25$ \\
\hline Gly & $142.75 \pm 41.69$ & $137.43 \pm 48.77$ & $114.79 \pm 39.37$ & $177.18 \pm 49.36$ & $153.20 \pm 64.06$ & $167.44 \pm 56.41$ \\
\hline Hсy & $8.36 \pm 0.87$ & $8.32 \pm 0.88$ & $7.97 \pm 0.60$ & $8.61 \pm 0.73$ & $8.91 \pm 1.24$ & $11.23 \pm 16.96$ \\
\hline His & $65.42 \pm 43.08$ & $47.99 \pm 19.87$ & $59.52 \pm 32.70$ & $86.02 \pm 60.47$ & $62.60 \pm 23.59$ & $93.33 \pm 82.02$ \\
\hline Leu & $95.90 \pm 26.36$ & $94.34 \pm 31.32$ & $74.40 \pm 22.21$ & $117.33 \pm 37.71$ & $104.91 \pm 35.82$ & $110.29 \pm 28.61$ \\
\hline Lys & $146.09 \pm 73.83$ & $112.32 \pm 67.79$ & $112.00 \pm 56.42$ & $115.47 \pm 56.26$ & $105.61 \pm 48.90$ & $139.96 \pm 95.51$ \\
\hline Met & $13.41 \pm 3.82$ & $14.04 \pm 4.00$ & $10.98 \pm 3.41$ & $16.46 \pm 6.48$ & $22.45 \pm 18.64$ & $17.31 \pm 5.99$ \\
\hline Orn & $15.24 \pm 7.63$ & $13.64 \pm 7.00$ & $14.58 \pm 11.25$ & $20.16 \pm 9.78$ & $16.41 \pm 4.29$ & $19.99 \pm 9.17$ \\
\hline Phe & $31.52 \pm 10.85$ & $29.74 \pm 7.20$ & $28.38 \pm 9.63$ & $35.56 \pm 9.15$ & $35.15 \pm 11.37$ & $47.97 \pm 60.92$ \\
\hline Piperine & $234.94 \pm 111.22$ & $211.58 \pm 96.55$ & $168.13 \pm 70.80$ & $165.37 \pm 51.62$ & $194.89 \pm 61.52$ & $189.45 \pm 92.27$ \\
\hline Pro & $403.66 \pm 153.75$ & $342.38 \pm 129.61$ & $297.51 \pm 106.06$ & $440.80 \pm 177.13$ & $471.58 \pm 109.39$ & $445.16 \pm 161.19$ \\
\hline Ser & $39.73 \pm 10.94$ & $39.94 \pm 13.49$ & $38.82 \pm 13.17$ & $65.50 \pm 28.25$ & $47.23 \pm 12.77$ & $54.44 \pm 21.33$ \\
\hline Thr & $23.04 \pm 7.95$ & $21.42 \pm 6.54$ & $19.63 \pm 4.86$ & $25.50 \pm 8.44$ & $24.07 \pm 5.85$ & $28.28 \pm 10.91$ \\
\hline Try & $41.18 \pm 10.33$ & $38.13 \pm 10.34$ & $40.84 \pm 11.36$ & $41.89 \pm 12.90$ & $37.86 \pm 12.11$ & $44.28 \pm 13.15$ \\
\hline Tyr & $39.62 \pm 10.86$ & $39.88 \pm 11.06$ & $35.02 \pm 10.24$ & $49.64 \pm 16.04$ & $45.63 \pm 19.91$ & $50.98 \pm 23.62$ \\
\hline Val & $108.13 \pm 26.37$ & $99.02 \pm 25.21$ & $95.52 \pm 23.72$ & $137.32 \pm 33.37$ & $128.29 \pm 37.53$ & $122.97 \pm 31.32$ \\
\hline $\mathrm{CO}$ & $27.29 \pm 8.21$ & $27.56 \pm 6.20$ & $26.12 \pm 13.30$ & $24.39 \pm 6.50$ & $23.86 \pm 6.26$ & $26.58 \pm 8.34$ \\
\hline $\mathrm{C} 2$ & $8.81 \pm 3.01$ & $8.20 \pm 2.64$ & $8.22 \pm 4.41$ & $8.99 \pm 3.41$ & $6.39 \pm 2.40$ & $8.30 \pm 3.02$ \\
\hline C3 & $1.09 \pm 0.49$ & $1.13 \pm 0.39$ & $1.02 \pm 0.41$ & $1.48 \pm 0.73$ & $0.98 \pm 0.22$ & $1.31 \pm 0.64$ \\
\hline $\mathrm{C} 3 \mathrm{DC}$ & $0.04 \pm 0.02$ & $0.04 \pm 0.01$ & $0.04 \pm 0.02$ & $0.04 \pm 0.02$ & $0.04 \pm 0.02$ & $0.04 \pm 0.02$ \\
\hline $\mathrm{C} 4$ & $0.15 \pm 0.07$ & $0.17 \pm 0.08$ & $0.20 \pm 0.14$ & $0.18 \pm 0.08$ & $0.14 \pm 0.04$ & $0.15 \pm 0.07$ \\
\hline C4DC & $0.49 \pm 0.35$ & $0.40 \pm 0.21$ & $0.48 \pm 0.19$ & $0.40 \pm 0.24$ & $0.48 \pm 0.24$ & $0.45 \pm 0.28$ \\
\hline $\mathrm{C} 4-\mathrm{OH}$ & $0.04 \pm 0.03$ & $0.04 \pm 0.02$ & $0.06 \pm 0.04$ & $0.06 \pm 0.03$ & $0.04 \pm 0.02$ & $0.05 \pm 0.04$ \\
\hline $\mathrm{C} 5$ & $0.10 \pm 0.04$ & $0.11 \pm 0.05$ & $0.10 \pm 0.05$ & $0.15 \pm 0.07$ & $0.13 \pm 0.05$ & $0.13 \pm 0.06$ \\
\hline C5:1 & $0.03 \pm 0.02$ & $0.04 \pm 0.02$ & $0.05 \pm 0.03$ & $0.04 \pm 0.03$ & $0.04 \pm 0.02$ & $0.05 \pm 0.03$ \\
\hline C5DC & $0.06 \pm 0.03$ & $0.06 \pm 0.03$ & $0.07 \pm 0.03$ & $0.06 \pm 0.04$ & $0.05 \pm 0.03$ & $0.05 \pm 0.03$ \\
\hline $\mathrm{C} 5-\mathrm{OH}$ & $0.17 \pm 0.07$ & $0.19 \pm 0.10$ & $0.22 \pm 0.11$ & $0.22 \pm 0.09$ & $0.24 \pm 0.09$ & $0.22 \pm 0.15$ \\
\hline C6 & $0.08 \pm 0.03$ & $0.08 \pm 0.03$ & $0.08 \pm 0.03$ & $0.07 \pm 0.03$ & $0.07 \pm 0.01$ & $0.07 \pm 0.03$ \\
\hline C6DC & $0.65 \pm 1.68$ & $0.35 \pm 0.16$ & $0.35 \pm 0.16$ & $0.33 \pm 0.11$ & $0.97 \pm 2.06$ & $0.55 \pm 0.76$ \\
\hline $\mathrm{C} 8$ & $0.08 \pm 0.05$ & $0.09 \pm 0.11$ & $0.07 \pm 0.03$ & $0.08 \pm 0.06$ & $0.05 \pm 0.03$ & $0.07 \pm 0.05$ \\
\hline $\mathrm{C} 10$ & $0.10 \pm 0.06$ & $0.11 \pm 0.10$ & $0.10 \pm 0.07$ & $0.08 \pm 0.06$ & $0.07 \pm 0.05$ & $0.08 \pm 0.07$ \\
\hline C10:1 & $0.07 \pm 0.04$ & $0.08 \pm 0.05$ & $0.07 \pm 0.03$ & $0.08 \pm 0.05$ & $0.06 \pm 0.03$ & $0.07 \pm 0.05$ \\
\hline $\mathrm{C} 10: 2$ & $0.52 \pm 0.27$ & $0.48 \pm 0.26$ & $0.53 \pm 0.20$ & $0.66 \pm 0.31$ & $0.51 \pm 0.25$ & $0.58 \pm 0.29$ \\
\hline
\end{tabular}


Supplementary Table 1. Continued

\begin{tabular}{|c|c|c|c|c|c|c|}
\hline Metabolites & NI & IFG & $\mathrm{SD}$ & DMVC & DPVD & DMVC \& DPVD \\
\hline $\mathrm{C} 12$ & $0.11 \pm 0.27$ & $0.06 \pm 0.03$ & $0.06 \pm 0.03$ & $0.05 \pm 0.03$ & $0.06 \pm 0.02$ & $0.08 \pm 0.13$ \\
\hline $\mathrm{C} 14$ & $0.07 \pm 0.06$ & $0.07 \pm 0.02$ & $0.06 \pm 0.03$ & $0.06 \pm 0.04$ & $0.06 \pm 0.03$ & $0.07 \pm 0.09$ \\
\hline C14:1 & $0.10 \pm 0.17$ & $0.09 \pm 0.06$ & $0.09 \pm 0.06$ & $0.06 \pm 0.03$ & $0.05 \pm 0.03$ & $0.07 \pm 0.05$ \\
\hline C14:2 & $0.37 \pm 0.28$ & $0.29 \pm 0.13$ & $0.03 \pm 0.02$ & $0.31 \pm 0.17$ & $0.43 \pm 0.53$ & $0.36 \pm 0.34$ \\
\hline C14DC & $0.02 \pm 0.02$ & $0.02 \pm 0.01$ & $0.34 \pm 0.17$ & $0.02 \pm 0.02$ & $0.03 \pm 0.02$ & $0.02 \pm 0.01$ \\
\hline $\mathrm{C} 14-\mathrm{OH}$ & $0.03 \pm 0.04$ & $0.03 \pm 0.02$ & $0.03 \pm 0.02$ & $0.03 \pm 0.01$ & $0.03 \pm 0.02$ & $0.03 \pm 0.02$ \\
\hline $\mathrm{C} 16$ & $0.71 \pm 0.33$ & $0.71 \pm 0.25$ & $0.75 \pm 0.34$ & $0.88 \pm 1.16$ & $0.68 \pm 0.26$ & $0.72 \pm 0.31$ \\
\hline $\mathrm{C} 16-\mathrm{OH}$ & $0.03 \pm 0.02$ & $0.03 \pm 0.02$ & $0.03 \pm 0.02$ & $0.03 \pm 0.03$ & $0.03 \pm 0.01$ & $0.05 \pm 0.09$ \\
\hline $\mathrm{C} 16: 1-\mathrm{OH}$ & $0.03 \pm 0.01$ & $0.04 \pm 0.02$ & $0.04 \pm 0.02$ & $0.04 \pm 0.02$ & $0.04 \pm 0.02$ & $0.05 \pm 0.02$ \\
\hline $\mathrm{C} 18$ & $0.46 \pm 0.16$ & $0.49 \pm 0.27$ & $0.47 \pm 0.23$ & $0.52 \pm 0.29$ & $0.43 \pm 0.17$ & $0.83 \pm 2.50$ \\
\hline C18:1 & $0.36 \pm 0.13$ & $0.37 \pm 0.11$ & $0.47 \pm 0.38$ & $0.40 \pm 0.18$ & $0.31 \pm 0.09$ & $0.34 \pm 0.14$ \\
\hline C18:2 & $0.99 \pm 0.36$ & $0.92 \pm 0.18$ & $0.98 \pm 0.37$ & $1.04 \pm 0.32$ & $0.90 \pm 0.23$ & $0.96 \pm 0.39$ \\
\hline $\mathrm{C} 18-\mathrm{OH}$ & $0.02 \pm 0.01$ & $0.02 \pm 0.02$ & $0.02 \pm 0.02$ & $0.02 \pm 0.02$ & $0.04 \pm 0.05$ & $0.02 \pm 0.02$ \\
\hline C18:1-OH & $0.00 \pm 0.01$ & $0.00 \pm 0.01$ & $0.00 \pm 0.01$ & $0.01 \pm 0.01$ & $0.03 \pm 0.07$ & $0.01 \pm 0.01$ \\
\hline $\mathrm{C} 20$ & $0.03 \pm 0.02$ & $0.03 \pm 0.02$ & $0.04 \pm 0.03$ & $0.03 \pm 0.02$ & $0.03 \pm 0.02$ & $0.03 \pm 0.03$ \\
\hline $\mathrm{C} 22$ & $0.05 \pm 0.02$ & $0.05 \pm 0.03$ & $0.06 \pm 0.03$ & $0.05 \pm 0.03$ & $0.05 \pm 0.02$ & $0.06 \pm 0.05$ \\
\hline $\mathrm{C} 24$ & $0.04 \pm 0.03$ & $0.04 \pm 0.02$ & $0.06 \pm 0.04$ & $0.05 \pm 0.02$ & $0.03 \pm 0.01$ & $0.05 \pm 0.05$ \\
\hline C26 & $0.03 \pm 0.01$ & $0.02 \pm 0.01$ & $0.03 \pm 0.02$ & $0.03 \pm 0.01$ & $0.02 \pm 0.01$ & $0.03 \pm 0.01$ \\
\hline
\end{tabular}

Values are presented as mean \pm standard deviation.

NI, normal individual; IFG, impaired fasting glucose; SD, simple diabetes; DMVC, diabetic microvascular complication; DPVD, diabetic peripheral vascular disease. 
Supplementary Table 2. Reference standards

\begin{tabular}{lc}
\hline Reference standard & Concentration, $\mathrm{nmol} / \mathrm{mL}$ \\
\hline${ }^{15} \mathrm{~N} ; 2$ - ${ }^{13} \mathrm{C}$-glycine & 12.5 \\
${ }^{2} \mathrm{H}_{4}$-alanine & 2.5 \\
${ }^{2} \mathrm{H}_{8}$-valine & 2.5 \\
${ }^{2} \mathrm{H}_{3}$-leucine & 2.5 \\
${ }^{2} \mathrm{H}_{3}$-methionine & 2.5 \\
${ }^{13} \mathrm{C}_{6}$-phenylananine & 2.5 \\
${ }^{13} \mathrm{C}_{6}$-tyrosine & 2.5 \\
${ }^{2} \mathrm{H}_{3}$-aspartate & 2.5 \\
${ }^{2} \mathrm{H}_{3}$-glutamate & 2.5 \\
${ }^{2} \mathrm{H}_{2}$-ornithine & 2.5 \\
${ }^{2} \mathrm{H}_{2}$-citrulline & 2.5 \\
${ }^{2} \mathrm{H}_{4}$; 5- ${ }^{13} \mathrm{C}$-arginine & 2.5 \\
${ }^{2} \mathrm{H}_{9}$-carnitine (free carnitine, CN) & 0.76 \\
${ }^{2} \mathrm{H}_{3}$-acetylcarnitine $(\mathrm{C} 2)$ & 0.19 \\
${ }^{2} \mathrm{H}_{3}$-propionylcarnitine $(\mathrm{C} 3)$ & 0.04 \\
${ }^{2} \mathrm{H}_{3}$-butyrylcarnitine $(\mathrm{C} 4)$ & 0.04 \\
${ }^{2} \mathrm{H}_{9}$-isovalerylcarnitine $(\mathrm{C} 5)$ & 0.04 \\
${ }^{2} \mathrm{H}_{3}$-octanoylcarnitine $(\mathrm{C} 8)$ & 0.04 \\
${ }^{2} \mathrm{H}_{9}$-myristoylcarnitine $(\mathrm{C} 14)$ & 0.04 \\
${ }^{2} \mathrm{H}_{3}$-palmitoylcarnitine $(\mathrm{C} 16)$ & 0.08 \\
\hline
\end{tabular}


Supplementary Table 3. Baselines characteristic of subjects

\begin{tabular}{|c|c|c|c|c|c|c|c|}
\hline Characteristic & $\begin{array}{c}\text { NI } \\
(n=54)\end{array}$ & $\begin{array}{c}\text { IFG } \\
(n=32)\end{array}$ & $\begin{array}{c}\mathrm{SD} \\
(n=21)\end{array}$ & $\begin{array}{c}\mathrm{DC} \\
(n=103)\end{array}$ & $\begin{array}{l}\text { DMVC } \\
(n=43)\end{array}$ & $\begin{array}{l}\text { DPVD } \\
(n=14)\end{array}$ & $P$ value \\
\hline Age, yr & $54.22 \pm 13.33$ & $41.69 \pm 11.70$ & $50.82 \pm 9.73$ & $55.78 \pm 9.23$ & $53.54 \pm 10.30$ & $57.08 \pm 8.04$ & $>0.05$ \\
\hline Duration of disease, $\mathrm{yr}$ & & & & $10.01 \pm 6.82$ & $8.65 \pm 6.68$ & $11.27 \pm 6.62^{\mathrm{a}}$ & $<0.05$ \\
\hline Male sex, $\%$ & 70.37 & 78.13 & 50.00 & 58.25 & 51.21 & 61.70 & $>0.05$ \\
\hline Body mass index, $\mathrm{kg} / \mathrm{m}^{2}$ & $22.86 \pm 3.21$ & $24.71 \pm 4.05^{\mathrm{a}}$ & $25.15 \pm 2.53^{\mathrm{a}}$ & $24.35 \pm 2.69^{\mathrm{a}}$ & $24.18 \pm 2.65^{\mathrm{a}}$ & $24.49 \pm 2.86^{\mathrm{a}}$ & $<0.05$ \\
\hline Systolic blood pressure, $\mathrm{mm} \mathrm{Hg}$ & $128.06 \pm 8.47$ & $126.75 \pm 10.50$ & $135.00 \pm 12.99^{\mathrm{a}}$ & $131.97 \pm 17.70^{\mathrm{a}}$ & $128.83 \pm 16.89$ & $134.73 \pm 18.19$ & $<0.05$ \\
\hline Diastolic blood pressure, $\mathrm{mm} \mathrm{Hg}$ & $81.87 \pm 7.90$ & $82.47 \pm 8.00$ & $89.86 \pm 11.77^{\mathrm{a}}$ & $80.37 \pm 10.06$ & $79.61 \pm 11.65$ & $80.57 \pm 9.02$ & $<0.05$ \\
\hline \multicolumn{8}{|l|}{ Laboratory data } \\
\hline Impaired fasting glucose, $\mathrm{mmol} / \mathrm{L}$ & & $6.49 \pm 0.23$ & $9.18 \pm 2.80^{\mathrm{b}}$ & $11.56 \pm 4.63^{b}$ & $13.90 \pm 5.80$ & $10.40 \pm 3.72$ & $<0.01$ \\
\hline HbAlc, \% & & & & $9.37 \pm 1.96$ & $10.04 \pm 1.96$ & $8.88 \pm 1.88^{\mathrm{b}}$ & $<0.01$ \\
\hline
\end{tabular}

Values are presented as mean \pm standard deviation.

NI, normal individual; IFG, impaired fasting glucose; SD, simple diabetes; DC, diabetic complication; DMVC, diabetic microvascular complication; DPVD, diabetic peripheral vascular disease; HbAlc, glycosylated hemoglobin.

${ }^{\mathrm{a}} \mathrm{P}<0.05,{ }^{\mathrm{b}} \mathrm{P}<0.01$. 
Supplementary Table 4. The parameters of differential metabolites of the other two cross-comparisons

\begin{tabular}{|c|c|c|c|c|c|}
\hline Metabolites & VIP & $P$ value & $P$ adjusted & $\log _{2}$ (fold change) & AUC \\
\hline \multicolumn{6}{|l|}{ SD vs. NI } \\
\hline C6DC & 2.064 & $4.10 \mathrm{E}-10$ & $2.38 \mathrm{E}-08$ & 3.701 & 0.986 \\
\hline Piperine & 2.015 & $4.22 \mathrm{E}-05$ & $2.45 \mathrm{E}-04$ & 1.079 & 0.819 \\
\hline $\mathrm{C} 12$ & 1.984 & $1.25 \mathrm{E}-08$ & $2.42 \mathrm{E}-07$ & 3.704 & 0.942 \\
\hline $\mathrm{C} 14: 1$ & 1.892 & $1.14 \mathrm{E}-08$ & $2.41 \mathrm{E}-07$ & 2.817 & 0.943 \\
\hline C4DC & 1.835 & 7.35E-07 & 7.10E-06 & 1.874 & 0.885 \\
\hline $\mathrm{C} 14: 2$ & 1.758 & $1.70 \mathrm{E}-06$ & $1.41 \mathrm{E}-05$ & 1.625 & 0.872 \\
\hline Gln & 1.719 & $4.20 \mathrm{E}-07$ & $4.88 \mathrm{E}-06$ & 1.177 & 0.894 \\
\hline $\mathrm{C} 14-\mathrm{OH}$ & 1.632 & $5.93 \mathrm{E}-08$ & $8.60 \mathrm{E}-07$ & 2.133 & 0.914 \\
\hline $\mathrm{C} 14$ & 1.612 & $1.16 \mathrm{E}-05$ & $7.50 \mathrm{E}-05$ & 2.047 & 0.840 \\
\hline \multicolumn{6}{|c|}{ DMVC vs. DPVD } \\
\hline Cys & 2.372 & $2.96 \mathrm{E}-04$ & $8.58 \mathrm{E}-03$ & -1.265 & 0.866 \\
\hline $\mathrm{C} 16$ & 2.209 & $3.32 \mathrm{E}-03$ & $2.75 \mathrm{E}-02$ & 2.801 & 0.797 \\
\hline Orn & 2.185 & $8.53 \mathrm{E}-04$ & $1.24 \mathrm{E}-02$ & 1.361 & 0.838 \\
\hline Ser & 2.052 & $2.12 \mathrm{E}-02$ & $9.38 \mathrm{E}-02$ & 1.212 & 0.734 \\
\hline $\mathrm{C} 24$ & 1.937 & $5.72 \mathrm{E}-04$ & $1.11 \mathrm{E}-02$ & 1.070 & 0.847 \\
\hline C6DC & 1.896 & $2.24 \mathrm{E}-05$ & $1.30 \mathrm{E}-03$ & -3.475 & 0.929 \\
\hline $\mathrm{C} 18: 1-\mathrm{OH}$ & 1.786 & $4.80 \mathrm{E}-02$ & $1.73 \mathrm{E}-01$ & -3.144 & 0.688 \\
\hline
\end{tabular}

VIP, variable importance in projection; AUC, area under curve; SD, simple diabetes; NI, normal individual; DMVC, diabetic microvascular complication; DPVD, diabetic peripheral vascular disease. 


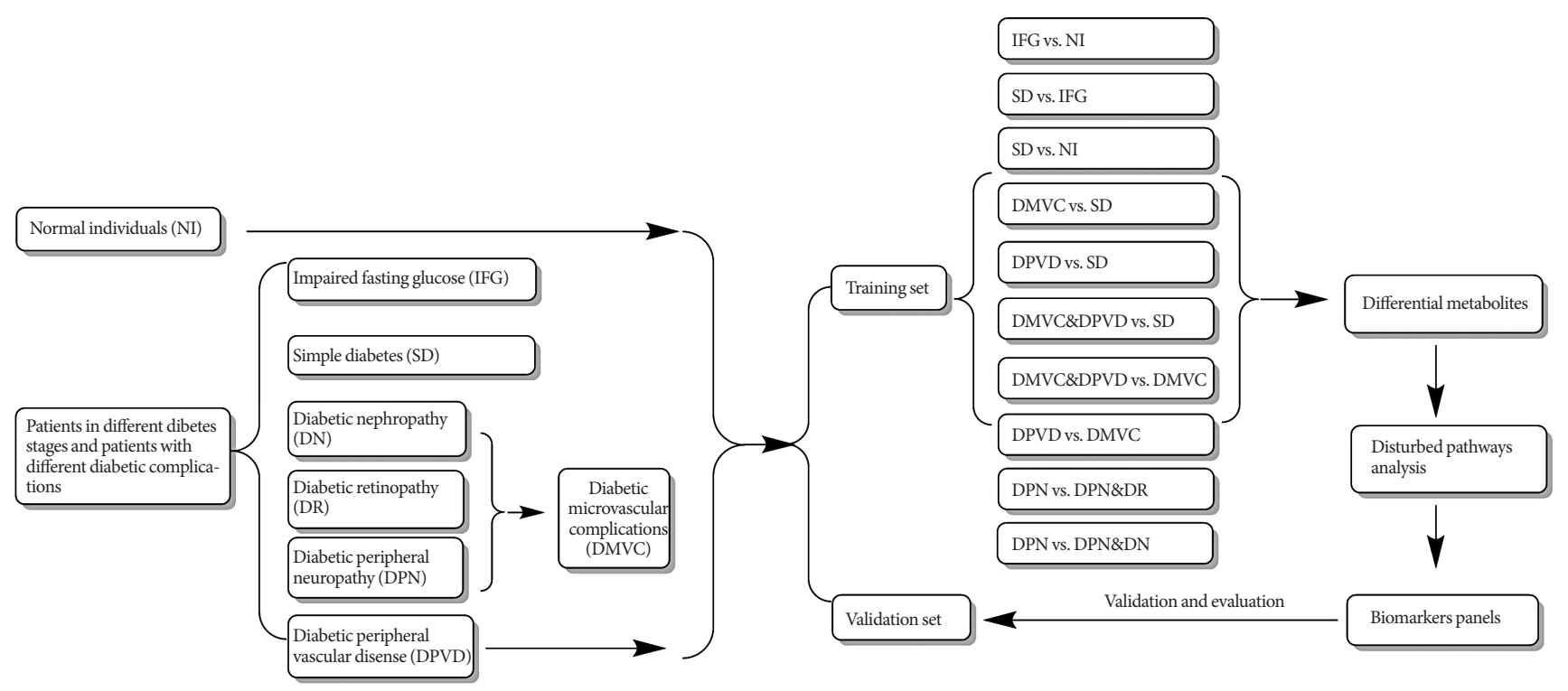

Supplementary Fig. 1. Study design. 

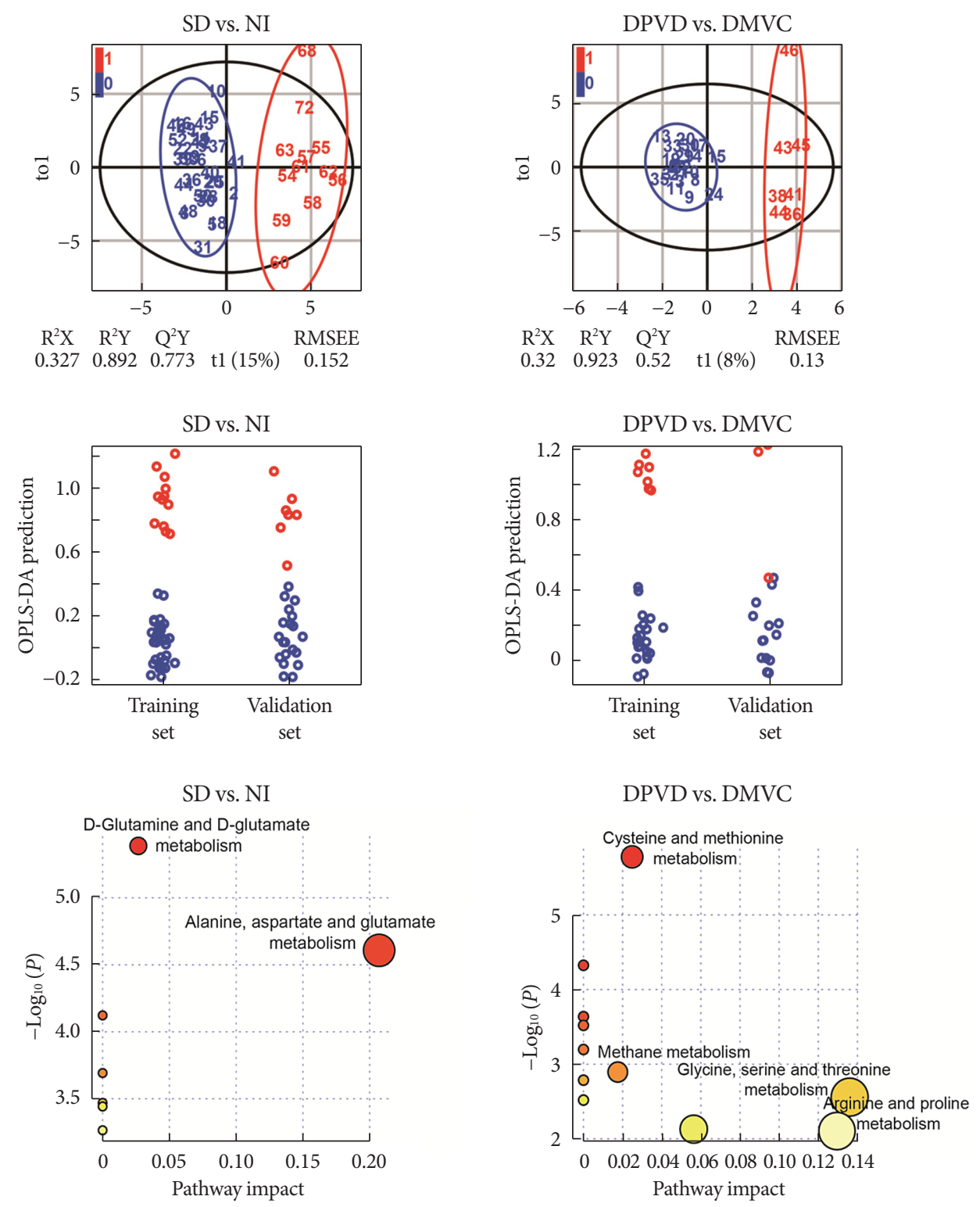

Supplementary Fig. 2. The score plot of orthogonal partial least squares discriminant analysis (OPLS-DA), prediction plots and differrtial metabolic pathways of the other two comparisons. NI, normal individual; SD, simple diabetes; DMVC, diabetic microvascular complication; DPVD, diabetic peripheral vascular disease. 